\title{
Role of the Gut Microbiome in the Pathophysiology and Treatment of Type 2 Diabetes Mellitus: A Mini Review
}

\author{
Anika Zainab, ${ }^{*}$, Arpit Mago², Asim Mehmood ${ }^{3}$, Mohamed Riad $^{4}$, Sneha Rajendra Sonavane \\ ${ }^{1}$ Department of Pathology, CMH Multan Institute of Medical Sciences, Multan, Pakistan. \\ ${ }^{2}$ Department of Clinical Medicine, Jawaharlal Nehru Medical College, Belgaum, India. \\ ${ }^{3}$ Department of Physiology, Shifa College of Medicine, Islamabad, Pakistan. \\ ${ }^{4}$ Department of Internal Medicine, Mansoura University, Mansoura, Egypt. \\ ${ }^{5}$ Department of Pathology, Grant Medical College \& Sir J.J. Hospital, Mumbai, India.
}

\begin{abstract}
Introduction: Diabetes mellitus type 2 is a chronic metabolic condition characterized by insulin resistance along with inadequate circulating insulin in the blood. It has been observed that the changes in the complex habitat composed of trillions of bacteria can cause metabolic disturbances. Previous studies have explained the phenomenon of dysbiosis which leads to the development and progression of diabetes mellitus type 2. It has been reported that there are altered gut microbiota levels present in patients with type 2 diabetes mellitus.
\end{abstract}

Main Body: Gut microbiota and its metabolites are involved in the pathogenesis of multiple metabolic diseases including type 2 diabetes mellitus therefore, intestinal microbiota may be one of the crucial medicinal targets for the disease. Studies have shown that diabetes can be managed effectively by altering the human gut microbiome. This review presents three therapeutic options which can be used to alter the gut microbiome. The therapeutic options are; fecal microbial transplant, use of probiotics and cinnamon. These interventions have proven to increase richness and diversity of gut microbiota. A vast number of studies support the beneficial effects of probiotics in treating type 2 diabetes due to its oxidative stress inhibiting properties. Cinnamon has shown similar benefits owing to its anti-inflammatory and anti-oxidative nature. An adequate number of animal studies and few human studies have reported improvement in insulin sensitivity in fecal microbial transplant recipients.

Conclusion: Consumption of probiotics and cinnamon have shown potential benefits in the treatment and prevention of type 2 diabetes mellitus. Fecal microbiota transplant has been shown to improve insulin sensitivity along with other impaired characteristics for example $\mathrm{HbA} 1 \mathrm{c}$ found in diabetics. It can be suggested that microbiota-based interventions can be used for the prevention and treatment of type 2 diabetes mellitus. Further studies are necessary to explore the mechanisms by which these treatment options provide potential beneficial effects in T2DM patients.

Keywords: Gut microbiome, Gut microbiota, Gut flora, Type 2 diabetes mellitus, Fecal microbial transplant, Probiotics.

\section{INTRODUCTION}

Diabetes mellitus type 2 (T2DM) is a chronic metabolic condition indicated by hyperglycemia, insulin resistance, and beta-cell dysfunction, with an increasing prevalence across the globe [1]. T2DM is a group of metabolic disorders characterized by decreased sensitivity of target organs to insulin which causes relative insufficiency of its secretion which is followed by protein, fat, water, electrolytes, and various other metabolic disorders [2]. Genetic predisposition determines an individual's susceptibility to T2DM, along with sedentary lifestyle and unhealthy way of living are crucial factors for the disease [3]. According to the latest (2017) estimates, the prevalence rate of T2DM was 6059 cases per 100,000. In the 2017 census, there were approximately 462 million people suffering from T2DM which accounts for $6.28 \%$ of the world's population [4]. Recent literature has observed that the

*Address correspondence to this author at the Department of Pathology, CMH Multan Institute of Medical Sciences, Multan, Pakistan.

Email: zainabanika50@gmail.com constitution of the intestinal microbiota can be a triggering cause of T2DM [5]. Gut microbiota is an intricate ecosystem of trillions of microorganisms that populate our gastrointestinal tract and has a pivotal role in modeling the physiology of the healthy host [6]. The intestinal microbiota profile may also be influenced by combination of different food and beverage we consume. Therefore, probiotics which are composed of useful microbes, alter the composition of intestinal microflora and control the metabolic parameters like insulin sensitivity [2]. There are 6 main phyla found in the human gut: Bacteroidetes, Firmicutes, Fusobacteria Actinobacteria, Verrucomicrobia, and Proteobacteria. The conformation of the gut microbiota is considered to be impacted by many factors, beginning from early life. During birth, the neonatal GIT is inhabited with microbes taking origin from maternal skin, fecal and vaginal flora [7]. The stability and diversity of the microbiota are influenced by environmental and genetic factors. An imbalance of these bacterial colonies is called dysbiosis that has been described in states of insulin resistance 
and obesity [8]. Consumption of foods high in fat content with low intake of dietary fibers and sugar has been suggested to cause the depletion of specific bacterial taxa along with a diverse group of the gut microbial community [9]. Dietary carbohydrates are the main source of energy for the gut microbiota. Fermentation of indigestible carbohydrates by these bacteria leads to the production of short-chain fatty acids (SCFAs), such as butyrate, acetate, and propionate [10]. SCFAs are a major energy source for both the host and the gut bacteria [11]. Recent studies found that a high-fat diet increases some gut bacteria species that generate high quantities of lipopolysaccharides that trigger the development of insulin resistance in hosts [12]. Altered intestinal microbiota was seen in T2DM patients which are depicted by a decrease in the Bacteroidetes/Firmicutes ratio and also an increase in multiple pathogens along with some endotoxins that produce gram-negative bacteria [13]. It was also seen that in obese individuals, intestinal microbiome was altered which can change the permeability of intestinal lining. It also increased the secretion of metabolic endotoxins (lipopolysaccharide/LPS). This may lead to persistent, low-grade inflammation impaired, insulin resistance, and T2DM [14]. New studies for the possible application of the gut microbiota in the treatment options for T2DM and the development of better prevention methods are emerging [15].

In this paper, we will review the role of gut microbiome in the pathogenesis of T2DM and look into the preventative and treatment strategies for metabolic health, insulin resistance, and T2DM.

\section{ROLE OF THE MICROBIOTA IN T2DM}

Gut microflora plays an important role in maintaining normal body homeostasis. Diabetes is related to an increase in inflammation, insulin resistance and deposition of adipose tissue, along with changes in the endocannabinoid system, intestinal peptide formation, leptin resistance that represent variations in the composition of the gut microbiome [16-18]. The incidence of type 2 diabetes mellitus depends on genetic as well as environmental factors. The composition and functionality of the gut microbiome are heavily influenced by diet and exercise. Studies done on mice have shown that when microbiota faces deleterious changes, the residual microbial component enters into circulation and leads to inflammation-induced insulin resistance $[19,20]$. Imbalance in the gut microbiome is associated with metabolic diseases like obesity and T2DM. In most patients, there is an alteration in the Firmicutes/Bacteroidetes ratio with an increase in Firmicutes and decreased Bacteroidetes [21, 22]. The microbiota of T2DM patients is also associated with decrease in butyrate-producing bacteria (especially Roseburia intestinalis and Faecalibacterium prausnitzii); moderate dysbiosis, increased oxidative stress, and reduced vitamin synthesis due to altered gene expression.
There are high levels of serum LPS concentration; and intestinal permeability [23].

The increase in Firmicutes is found to be associated with a poorer metabolism, decrease levels of glycan-degrading enzymes, and a reduction in energy expenditure at the resting state [24]. The fall in Bacteroidetes levels causes a decrease in acetate and propionate production. But the decrease in butyrate formation and its producing species is significantly linked to diabesity [25]. Butyrate acts on the gut-brain axis and works via the vagus nerve to reduce insulin sensitivity, improve metabolism and reduce appetite. Both propionates as well as butyrate stimulate intestinal gluconeogenesis and help maintain glucose homeostasis and energy levels [26]. Dysbiosis and its negative interaction with genetic and environmental factors induce altered immune response leading to development or deterioration of T2DM [27]. The lipopolysaccharide in the wall of microbes activates toll-like receptors and induces inflammatory cytokines to be released by stimulation of the immune system. LPS promotes insulin resistance and the insulin signaling deficiency in the muscle, adipose tissue, liver, and hypothalamus by activation of nuclear factor kappa-B and c-Jun N-terminal kinase pathways [28]. The gut biota also produces small chain fatty acids (SCFA). Butyrate type of SCFA improves insulin sensitivity and secretion by stimulation of peptide 1 like glucagon (GLP-1) and reduction of adipocyte inflammation [29]. The role of gut microbiota in bile acid metabolism is equally important. They modulate the bile acid signaling via the farnesoid X receptor (FXR) and the G protein-coupled membrane receptor 5 (TGR5), responsible for the regulation of various host metabolic pathways FXR and TGR5 signaling regulates glucose homeostasis by controlling glucagon-like peptide-1 (GLP-1) production in intestinal L cells $[30,31]$. Hence efforts must be made at improving it by different interventions including consumption of probiotics, lifestyle modifications, and fecal micro transplants.

\section{THERAPEUTIC APPLICATION OF MICROBIOME IN T2DM}

\section{Probiotics}

Several meta-analyses have shown a reduction in glucose, insulin, HbA1C as well as HOMA-IR in participants with diabetes post probiotic use [32]. HOMA-IR (Homeostasis Model Assessment of Insulin Resistance) is a tool which measures IR (Insulin Resistance) quantitatively. It is also used to assess beta cell function. Metthews et al. introduced this tool in 1985 [33]. Whilst the exact mechanism of action of probiotics is not clear, there are many proposed mechanisms. The increase in lactic acid with the use of probiotics has been found to reduce intestinal glucose uptake. Besides they can also improve intestinal barrier function, SCFA production, and regulation of bile acid metabolism preventing and delay- 
ing pancreatic degradation [34]. Studies have also reported use of probiotics in conjunction with an increase in hepatic glycogen causes suppression PEPCK and G-6-Phosphatase which are rate limiting enzymes of gluconeogenesis [35]. A recent meta-analysis of 13 randomized control trials involving 840 subjects with T2DM concluded that probiotics show significant reduction in serum fasting blood glucose levels and there was a non-significant effect on HbAlc. It has been proposed that probiotics remove oxidative stress and the phenomenon of oxidative stress is well established in the pathogenesis of T2DM [36]. Though some studies indicate limited benefits by the use of probiotics in diabetes treatment, a vast majority of the studies included report its benefits [37, 38]. This may be due to variations in strains used in different probiotics and hence warrants a more precise study on different probiotics used.

\section{Fecal Microbiota Transplant (FMT)}

It is the procedure of the transfer of fecal microbes from a healthy donor to a recipient. It is successful in treating Clostridium difficile infection, besides other metabolic diseases. A recent study by Vrieze et al. has demonstrated improvements in insulin sensitivity in the periphery and an increase in butyrate-producing bacteria in the recipient's fecal microbiota post-FMT from a lean donor to an adult male with metabolic syndrome [39]. A significant improvement of intestinal microbiota with reduction of plasma glycolipid levels has been found in $\mathrm{db} / \mathrm{fb}$ mice with the use of individuals with normal glucose tolerance. An increase in the level of A. muciniphila was found to correlate with HDAC3 expression [40].

\section{Role of Cinnamon}

Cinnamon has been reported to reduce average fasting blood glucose levels, HbAlc as well as insulin resistance in intervention groups in comparison with the control groups $[41,42]$.

The commercial value of cinnamon is determined by cinnamaldehyde. It has been found to have anti-inflammatory, anti-oxidant, anti-microbial and anti-spasmodic qualities. Cinnamon exerts an inhibitory action on the Helicobacter pylori damaging effect and also inhibits the growth of Escherichia coli, Staphylococcus aureus, Salmonella Typhimurium, Listeria monocytogenes and Clostridium species [43]. There is reduction in the phosphorylation of insulin receptor in T2DM patients. Compounds in the cinnamon increase insulin sensitivity by stimulation of insulin-receptor autophosphorylation and inhibiting phosphotyrosine phosphatase. Many studies have indicated an increase in glucose uptake and glycogen synthesis by stimulation of insulin receptors [44]. A study consisted of 136 participants, 30 to 40 years of age, suffering from type 2 diabetes mellitus were divided into four groups; probiotics group, cinnamon group, probiotics and cinnamon group and control group. There was no significant difference seen in the serum glucose levels or $\mathrm{HbA} 1 \mathrm{c} \%$ in initial stages of the trial. After three months of treatment, a significant decrease in fasting blood glucose was observed when compared with the control group. The mean HbA1c \% also declined in the three groups as compared with the control group [45].

\section{CONCLUSION}

We have concluded that alteration in gut microbiota causes insulin resistance which ultimately leads to a metabolic disorder like Diabetes Mellitus Type 2. It has been observed that there is an alteration in Firmicutes/Bacteroidetes ratio with an increase in Firmicutes and decreased Bacteroidetes with various metabolites and toxins produced by these bacteria which are responsible for the pathogenesis of T2DM. We have discussed some preventative and treatment options for T2DM i.e. probiotics, fecal microbiota transplant and cinnamon. These interventions can improve the prognosis of this disorder and improve patients' quality of life suffering from this disorder. Further studies are required to establish mechanisms by which these treatment options work.

\section{LIST OF ABBREVIATIONS}

FMT: Fecal Microbiota Transplant.

FXR: Farnesoid X Receptor.

HDCAC3: Histone Deacetylases 3.

IR: Insulin Resistance.

HOMA-IR: Homeostatic Model Assessment for Insulin Resistance.

LPS: Lipopolysaccharides.

PEPCK: Phosphoenolpyruvate Carboxykinase.

SCFAs: Short-Chain Fatty Acids.

T2DM: Diabetes Mellitus Type 2.

\section{AUTHORS' CONTRIBUTION}

Anika Zainab, Mohamed Riad: Conception and ideation, Literature review and Data collection.

Anika Zainab, Arpit Mago, Asim Mehmood, Sneha Rajendra Sonavane: Drafting the article.

Anika Zainab, Arpit Mago, Asim Mehmood: Critical Revision of the article.

Anika Zainab, Mohamed Riad, Arpit Mago, Asim Mehmood, Sneha Rajendra Sonavane: Final Approval of the revision to be published.

\section{CONFLICT OF INTEREST}

Declared none.

\section{ACKNOWLEDGEMENTS}

Declared none. 


\section{REFERENCES}

[1] Horvath A, Leber B, Feldbacher N. Effects of a multispecies synbiotic on glucose metabolism, lipid marker, gut microbiome composition, gut permeability, and quality of life in diabesity: A randomized, double-blind, placebo-controlled pilot study. Eur J Nutr 2020; 59(7): 2969-83. DOI: 10.1007/s00394-019-02135-w

[2] Ma Q, Li Y, Li P, et al. Research progress in the relationship between type 2 diabetes mellitus and intestinal flora. Biomed Pharmacother 2019; 117: 109138. DOI: 10.1016/j.biopha.2019.109138

[3] Zheng Y, Ley SH, Hu FB. Global aetiology and epidemiology of type 2 diabetes mellitus and its complications. Nat Rev Endocrinol 2018; 14(2): 88-98. DOI: 10.1038/nrendo. 2017.151

[4] Khan MAB, Hashim MJ, King JK, Govender RD, Mustafa H, Al Kaabi J. Epidemiology of type 2 diabetes - global burden of disease and forecasted trends. J Epidemiol Glob Health 2020; 10(1): 107-11. DOI: 10.2991/jegh.k.191028.001

[5] Salgaço MK, Oliveira LGS, Costa GN, Bianchi F, Sivieri K. Relationship between gut microbiota, probiotics, and type 2 diabetes mellitus. Appl Microbiol Biotechnol 2019; 103(23-24): 9229-38. DOI: 10.1007/s00253-019-10156-y

[6] Fernández-Veledo S, Vendrell J. Gut microbiota-derived succinate: Friend or foe in human metabolic diseases? Rev Endocr Metab Disord 2019; 20(4): 439-47. DOI: 10.1007/s11154-019-09513-z

[7] Zhu T, Goodarzi MO. Metabolites linking the gut microbiome with risk for type 2 diabetes. Curr Nutr Rep 2020; 9(2): 83-93. DOI: $10.1007 / \mathrm{s} 13668-020-00307-3$

[8] Salles BIM, Cioffi D, Ferreira SRG. Probiotics supplementation and insulin resistance: A systematic review. Diabetol Metab Syndr 2020; 12(1): 98. DOI: 10.1186/s13098-020-00603-6

[9] Ojo O, Feng QQ, Ojo OO, Wang XH. The role of dietary fiber in modulating gut microbiota dysbiosis in patients with type 2 diabetes: A systematic review and meta-analysis of randomised controlled trials. Nutrients 2020; 12(11): 3239. DOI: $10.3390 /$ nu12113239

[10] Koh A, De Vadder F, Kovatcheva-Datchary P, Bäckhed F. From dietary fiber to host physiology: Short-chain fatty acids as key bacterial metabolites. Cell 2016; 165(6): 1332-45. DOI: 10.1016/j.cell.2016.05.041

[11] Portune KJ, Beaumont M, Davila A-M, Tomé D, Blachier F, Sanz Y. Gut microbiota role in dietary protein metabolism and health-related outcomes: The two sides of the coin. Trends Food Sci Technol 2016; 57: 213-32. DOI: 10.1016/j.tifs.2016.08.011
[12] Yao K, Zeng L, He Q, Wang W, Lei J, Zou X. Effect of probiotics on glucose and lipid metabolism in type 2 diabetes mellitus: A meta-analysis of 12 randomized controlled trials. Med Sci Monit 2017; 23: 3044-53. DOI: 10.12659/MSM.902600

[13] Woldeamlak B, Yirdaw K, Biadgo B. Role of gut Microbiota in type 2 diabetes mellitus and its complications: Novel insights and potential intervention strategies. Korean J Gastroenterol 2019; 74(6): 314-20. DOI: 10.4166/kjg.2019.74.6.314

[14] Navab-Moghadam F, Sedighi M, Khamseh ME, et al. The association of type II diabetes with gut microbiota composition. Microb Pathog 2017; 110: 630-6. DOI: 10.1016/j.micpath.2017.07.034

[15] Brunkwall L, Orho-Melander M. The gut microbiome as a target for prevention and treatment of hyperglycaemia in type 2 diabetes: From current human evidence to future possibilities. Diabetologia 2017; 60(6): 943-51. DOI: $10.1007 / \mathrm{s} 00125-017-4278-3$

[16] Dang F, Jiang Y, Pan R, et al. Administration of Lactobacillus paracasei ameliorates type 2 diabetes in mice. Food Funct 2018; 9(7): 3630-9. DOI: 10.1039/C8FO00081F

[17] Kassaian N, Aminorroaya A, Feizi A, Jafari P, Amini M. The effects of probiotic and synbiotic supplementation on metabolic syndrome indices in adults at risk of type 2 diabetes: Study protocol for a randomized controlled trial. Trials 2017; 18(1): 148. DOI: $10.1186 / \mathrm{s} 13063-017-1885-8$

[18] Cani PD, Delzenne NM. The role of the gut microbiota in energy metabolism and metabolic disease. Curr Pharm Des 2009; 15(13): 1546-58. DOI: 10.2174/138161209788168164

[19] Cani PD, Bibiloni R, Knauf C, et al. Changes in gut microbiota control metabolic endotoxemia-induced inflammation in high-fat diet-induced obesity and diabetes in mice. Diabetes 2008; 57(6): 1470-81. DOI: 10.2337/db07-1403

[20] Cani PD, Amar J, Iglesias MA, et al. Metabolic endotoxemia initiates obesity and insulin resistance. Diabetes 2007; 56(7): 1761-72. DOI: 10.2337/db06-1491

[21] Chobot A, Górowska-Kowolik K, Sokołowska M, Jarosz-Chobot P. Obesity and diabetes-Not only a simple link between two epidemics. Diabetes Metab Res Rev 2018; 34(7): e3042. DOI: $10.1002 /$ dmrr.3042

[22] Le Chatelier E, Nielsen T, Qin J, et al. Richness of human gut microbiome correlates with metabolic markers. Nature 2013; 500(7464): 541-6. DOI: 10.1038/nature12506

[23] Roager HM, Vogt JK, Kristensen M, et al. Whole grain-rich diet reduces body weight and systemic low-grade inflammation without inducing major changes of the gut microbiome: A randomised cross-over trial. Gut 2019; 68(1): 83-93. DOI: 10.1136/gutjnl-2017-314786

[24] Ortega MA, Fraile-Martínez O, Naya I, et al. Type 2 diabetes 
mellitus associated with obesity (diabesity). The central role of gut Microbiota and its translational applications. Nutrients 2020; 12(9): 2749. DOI: 10.3390/nu12092749

[25] Johnson EL, Heaver SL, Walters WA, Ley RE. Microbiome and metabolic disease: Revisiting the bacterial phylum Bacteroidetes. J Mol Med 2017; 95(1): 1- 8. DOI: 10.1007/s00109-016-1492-2

[26] Li Z, Yi CX, Katiraei S, et al. Butyrate reduces appetite and activates brown adipose tissue via the gut-brain neural circuit. Gut 2018; 67(7): 1269-79. DOI: 10.1136/gutjnl-2017-314050

[27] Razmpoosh E, Javadi A, Ejtahed HS, Mirmiran P, Javadi M, Yousefinejad A. The effect of probiotic supplementation on glycemic control and lipid profile in patients with type 2 diabetes: A randomized placebo controlled trial. Diabetes Metab Syndr 2019; 13(1): 175-82. DOI: 10.1016/j.dsx.2018 .08 .008

[28] Newsholme P, Cruzat VF, Keane KN, Carlessi R, de Bittencourt PIH Jr. Molecular mechanisms of ROS production and oxidative stress in diabetes. Biochem J 2016; 473(24): 4527-50. DOI: 10.1042/BCJ20160503C

[29] Ríos-Covián D, Ruas-Madiedo P, Margolles A, Gueimonde M, de Los Reyes-Gavilán CG, Salazar N. Intestinal short chain fatty acids and their link with diet and human health. Front Microbiol 2016; 7: 185. DOI: 10.3389/fmicb.2016.00185

[30] Trabelsi M-S, Daoudi M, Prawitt J, et al. Farnesoid X receptor inhibits glucagon-like peptide-1 production by enteroendocrine L cells. Nat Commun 2015; 6: 7629. DOI: 10.1038/ncomms 8629

[31] Thomas C, Gioiello A, Noriega L, et al. TGR5-mediated bile acid sensing controls glucose homeostasis. Cell Metab 2009; 10(3): 167-77. DOI: 10.1016/j.cmet.2009.08.001

[32] Sun J, Buys NJ. Glucose- and glycaemic factor-lowering effects of probiotics on diabetes: A meta-analysis of randomised placebo-controlled trials. Br J Nutr 2016; 115(7): 1167-77. DOI: $10.1017 / \mathrm{S} 0007114516000076$

[33] Matthews DR, Hosker JP, Rudenski AS, Naylor BA, Treacher DF, Turner RC. Homeostasis model assessment: Insulin resistance and beta-cell function from fasting plasma glucose and insulin concentrations in man. Diabetologia 1985; 28: 412- 9. DOI: 10.1007/BF00280883

[34] Javanmard A, Ashtari S, Sabet B. Probiotics and their role in gastrointestinal cancers prevention and treatment: An overview. Gastroenterol Hepatol Bed Bench 2018; 11(4): 284-95.

[35] Lee CB, Chae SU, Jo SJ, Jerng UM, Bae SK. The relationship between the gut microbiome and metformin as a key for treating type 2 diabetes mellitus. Int J Mol Sci 2021; 22(7): 3566. DOI: $10.3390 /$ ijms 22073566

[36] Ardeshirlarijani E, Tabatabaei-Malazy O, Mohseni S, Qorbani M, Larijani B, Baradar Jalili R. Effect of probiotics supplementation on glucose and oxidative stress in type 2 diabetes mellitus: A meta-analysis of randomized trials. Daru 2019; 27(2): 827-37. DOI: 10.1007/s40199-019-00302-2

[37] Dong Y, Xu M, Chen L, Bhochhibhoya A. Probiotic foods and supplements interventions for metabolic syndromes: A systematic review and meta-analysis of recent clinical trials. Ann Nutr Metab 2019; 74(3): 224-41. DOI: 10.1159/000499028

[38] Ruan Y, Sun J, He J, Chen F, Chen R, Chen H. Effect of probiotics on glycemic control: A systematic review and meta-analysis of randomized, controlled trials. PLoS One 2015; 10(7): e0132121. DOI: 10.1371/journal.pone.0132121

[39] Vrieze A, Van Nood E, Holleman F, et al. Transfer of intestinal microbiota from lean donors increases insulin sensitivity in individuals with metabolic syndrome. Gastroenterology 2012; 143(4): 913-6.e7. DOI: 10.1053/j.gastro.2012.06.031

[40] Zhang P-P, Li L-L, Han X, et al. Fecal microbiota transplantation improves metabolism and gut microbiome composition in $\mathrm{db} / \mathrm{db}$ mice. Acta Pharmacol Sin 2020; 41(5): 678-85. DOI: 10.1038/s41401-019-0330-9

[41] Lee DK, Jang S, Baek EH, et al. Lactic acid bacteria affect serum cholesterol levels, harmful fecal enzyme activity, and fecal water content. Lipids Health Dis 2009; 8: 21. DOI: 10.1186/1476-511X-8-21

[42] Mang B, Wolters M, Schmitt B, et al. Effects of a cinnamon extract on plasma glucose, $\mathrm{HbA}$, and serum lipids in diabetes mellitus type 2. Eur J Clin Invest 2006; 36(5): 340-4. DOI: 10.1111/j.1365-2362.2006.01629.x

[43] Liu Q, Meng X, Li Y, Zhao C-N, Tang G-Y, Li H-B. Antibacterial and antifungal activities of spices. Int J Mol Sci 2017; 18(6): 1283. DOI: 10.3390/ijms 18061283

[44] Medagama AB. The glycaemic outcomes of Cinnamon, a review of the experimental evidence and clinical trials. Nutr J 2015; 14: 108. DOI: 10.1186/s12937-015-0098-9

[45] Mirmiranpour H, Huseini HF, Derakhshanian H, Khodaii Z, Tavakoli-Far B. Effects of probiotic, cinnamon, and synbiotic supplementation on glycemic control and antioxidant status in people with type 2 diabetes; a randomized, double-blind, placebo-controlled study. J Diabetes Metab Disord 2019; 19(1): 53-60. DOI: 10.1007/s40200-019-00474-3 